\title{
Geradores de estresse para os trabalhadores de enfermagem de centro cirurgico
}

\author{
Stress Generators for employees of surgical nursing center
}

\author{
João Paulo Belini Jacques ${ }^{1}$; Renata Perfeito Ribeiro²; Julia Trevisan Martins ${ }^{3}$; \\ Danilo Servilha Rizzi4; Denise Rodrigues Costa Schmidt ${ }^{5}$
}

\begin{abstract}
Resumo
O objetivo deste estudo foi identificar os fatores que contribuem para o estresse entre trabalhadores de enfermagem de Centro Cirúrgico de um hospital publico de grande porte e alta complexidade. Pesquisa descritiva de natureza qualitativa, realizada em um Centro Cirúrgico de um hospital Universitário do estado do Paraná. Os dados foram coletados entre os meses de agosto e setembro de 2013, por meio de entrevista semiestruturada com 15 membros da equipe de enfermagem. Aplicou-se análise de conteúdo temática resultando em três categorias: sobrecarga de trabalho; falta de planejamento, de recursos humanos, de materiais e equipamentos; e ambiente fechado. Conclui-se que os profissionais de enfermagem vivenciaram o estresse relacionado com fatores de natureza objetiva como a sobrecarga de trabalho, a falta de planejamento das atividades, dos recursos humanos e dos materiais e equipamentos e o viver em um ambiente fechado.
\end{abstract}

Palavras-chave: Estresse. Centro cirúrgico. Equipe de enfermagem.

\begin{abstract}
The aim of this study was to identify factors that contribute to stress among workers in a surgical center. Descriptive qualitative research, performed in a surgery center of a university hospital in the state of Paraná. Data were collected between the months of August and September 2013, through semi-structured interviews with 15 members of the nursing team. Applied if the thematic content analysis resulting in three categories: work overload; lack of planning, human resources, materials and equipment and confinement. It is concluded that nursing professionals have experienced work related stress factors of objective nature such as work overload, lack of activity planning, human resources and materials and equipment and living in a closed environment.
\end{abstract}

Keywords: Stress. Surgical center. Nursing staff.

${ }^{1}$ Residente de enfermagem Perioperatória do Departamento de Enfermagem da Universidade Estadual de Londrina.

${ }^{2}$ Doutora. Docente do Departamento de Enfermagem da Universidade estadual de Londrina. Docente do Programa de Mestrado em Enfermagem da Universidade Estadual de Londrina.

${ }^{3}$ Doutora. Docente do Departamento de Enfermagem da Universidade estadual de Londrina. Docente do Programa de Mestrado em Enfermagem da Universidade Estadual de Londrina.

${ }^{4}$ Enfermeiro do Hospital São Camilo: Hospital Cura D’ars. Fortaleza-CE.

${ }^{5}$ Doutora. Enfermeira da Sala de Recuperação Anestésica do Centro Cirúrgico do Hospital Universitário de Londrina. 


\section{Introdução}

O Centro Cirúrgico (CC) apresenta-se como um setor com características de trabalho diferenciadas dos demais, o que pode contribuir para o estresse entre os trabalhadores da área da saúde em especial os da enfermagem, que são aqueles que realizam a assistência ao paciente cirúrgico, além do suporte aos demais membros da equipe cirúrgica.

Essas circunstâncias estressantes do trabalho da enfermagem podem estar ligadas ao ambiente laboral do CC por se apresentar como um setor fechado com sobrecarga de trabalho em proporção inadequada ao número de trabalhadores de enfermagem qualificados, turnos rotativos, baixa remuneração, risco biológico inerente à manipulação de pacientes em período perioperatório, o risco físico determinado pelo uso de Raios-X nas salas cirúrgicas, o trabalho em postura inadequada em pé e o uso de novas tecnologias sem o devido preparo (OLER et al., 2005; SANTOS; BERESIN, 2009).

Os sentimentos de frustração e descontentamento em relação à responsabilidade e exercício profissional dos trabalhadores da enfermagem constituem-se como situações geradoras de transtornos físicos, psicológicos e sociais podendo afetar a saúde desses trabalhadores com o comprometimento da sua qualidade de vida, além de interferir nas relações de trabalho e no desempenho das suas atividades laborais (OLER et al., 2005; SEMENIUK; DURMAN; MATOS 2012), colocando-os em uma escala de alto grau de estresse (RIBEIRO, 2012).

O estresse é resultante da percepção da discordância entre as exigências de determinadas tarefas e a presença de recursos pessoais para cumpri-las. No trabalho, o estresse pode refletir-se em atrasos, absenteísmo, insatisfação, sabotagem, baixos níveis de desempenho e até mesmo violência no local de trabalho (MONTANHOLI; TAVARES; OLIVEIRA, 2006; RODRIGUES; CHAVES,
2008). Na falta de interferências que minimizem essa situação, os trabalhadores podem sentir-se exauridos, sem energia e depressivos, podendo desenvolver doenças como síndrome metabólica, síndrome da fadiga crônica, distúrbios do sono, diabetes e a síndrome de Burnout (SCHMIDT et al., 2009; RIBEIRO, 2012). Quanto mais avançada a fase de estresse, mais doenças e agravos à saúde o individuo pode apresentar (PASSOS; SILVA; CARVALHO, 2010).

Ressalta-se que a responsabilidade e qualificação exigida dos trabalhadores do CC atreladas ao excesso de atividades, aos recursos humanos insuficientes e a falta de equipamentos ou materiais necessários à assistência prestada ao paciente cirúrgico, acarretam ao longo dos anos desgaste físico, emocional e social. Assim, os serviços de saúde devem implementar medidas para minimizar o estresse no trabalho, dentre essas, destacam-se o apoio aos profissionais, promover a interação multiprofissional, a melhoria das condições de trabalho e da infraestrutura da unidade, investir em programas de atenção à saúde do trabalhador, organização racional e valorização do trabalho (BRANDÃO; GALVÃO, 2013).

Schmidt etal.(2009) afirmam que é fundamental a realização de estudos direcionados a esse grupo de trabalhadores, visto que o estresse está presente no seu cotidiano, resultante de inúmeros fatores relacionados ao tipo de ambiente, complexidade das relações humanas e de trabalho, autonomia profissional, grau elevado de exigência quanto às competências e habilidades, alta responsabilidade e planejamento adequado de recursos humanos e materiais.

A interação do indivíduo ocorre quando o mesmo age, percebe, interpreta e volta a agir, de modo que se torna um ator dinâmico em constante processo de socialização e interação com o meio e com os outros e não uma personalidade estruturada e imutável (CHARON, 1989). Assim, 
o Interacionismo Simbólico centra-se na interação e na dinâmica das atividades sociais que ocupam o espaço entre os indivíduos, e são a causa do comportamento deles, ou seja, a ação dos atores é derivada da significação; essa significação deriva ou origina-se das interações sociais; por fim, as significações são empregadas pelos atores sociais nas interações sociais grupais, que por sua vez, modificam as próprias significações (DUPAS; OLIVEIRA; COSTA, 1997).

Assim sendo, este estudo teve como objetivo identificar os fatores que contribuem para o estresse dos trabalhadores do CC de um hospital público de grande porte.

\section{Material e Método}

Pesquisa qualitativa, realizada com a equipe de enfermagem de um CC de um hospital universitário do estado do Paraná.

Adotaram-se como critérios de inclusão: ambos os sexos e estar trabalhando na referida unidade há no mínimo dois anos. Foram excluídos os que se encontravam de férias ou licença. Enfatiza-se que houve intencionalidade ao escolher-se os participantes da pesquisa, porém a definição do número de sujeitos não se baseou em critérios de representatividade numérica. Assim, não se definiu um tamanho amostral, pois as entrevistas foram realizadas até ocorrer à convergência das falas, em relação ao fenômeno estudado, totalizando 15 entrevistados (MINAYO, 2010).

A coleta de dados foi realizada no próprio local do trabalho, em sala reservada e horários pré-agendados, nos meses de agosto e setembro de 2013, por meio de entrevistas individuais e gravadas. As entrevistas foram realizadas utilizando-se um roteiro semiestruturado com a seguinte questão norteadora: Que fatores você considera que provocam estresse no seu cotidiano laboral?
Para tratamento dos dados utilizou-se a técnica de Análise de Conteúdo, na modalidade temática com as etapas: leitura, determinação das unidades de registro e significações, codificação e classificação; tratamento e interpretação dos resultados obtidos. Por meio da leitura flutuante, foi possível proceder com as marcações das unidades de registro, organizando-as por temas. Por meio de aproximações e distanciamento construíram-se as categorias (BARDIN, 2011).

Adotou-se como referencial teórico o Interacionismo Simbólico, visto que as ideias centrais baseiam-se no processo de interação, em que os indivíduos são ativos e aprendem a dar significado às coisas. Os participantes da pesquisa foram identificados utilizando-se as letras E1, E2, E3 e, assim, sucessivamente, para a apresentação de suas falas e preservação de seu anonimato.

A pesquisa foi aprovada pelo Comitê de Ética em Pesquisa Envolvendo Seres Humanos da Universidade Estadual de Londrina (UEL) com CAAE $n^{\circ}$ 0283.0.268.000-11.

\section{Resultados e Discussão}

Os participantes desta pesquisa foram todos do gênero feminino, com faixa etária compreendida entre 33 e 59 anos, nove casadas, três solteiras, duas divorciadas e uma viúva, sendo três enfermeiras, oito técnicas de enfermagem e quatro auxiliares de enfermagem, com um tempo de trabalho variando de 2 a 23 anos em centro cirúrgico (média de 10,6 anos).

A análise das falas levou a construção de três categorias temáticas: Sobrecarga de trabalho; Falta de planejamento das atividades, dos recursos humanos e materiais; e Ambiente fechado;

Em relação à categoria denominada de Sobrecarga de trabalho apresentam-se as seguintes falas: 
Devido as intercorrências e as urgências que têm, são comuns, fica muito corrido, você não tem tempo para fazer suas tarefas com o tempo que deveria, com o tempo certo e ai você tem que fazer a mais, além da sua capacidade e daí é aquela sobrecarga é aquele estresse. (E1)

Na maioria das vezes a gente está sobrecarregada. É assim: quando você chega e vê o planinho de trabalho e nota que foi escalada para cirurgias que não estava escalada e, você tem que instrumentar de novo isso provoca um estresse danado. (E6)

Se tiver muita cirurgia de manhã já passa muita cirurgia para tarde, ai já é mais complicado para quem está à tarde, ai já pega o bonde andando, já é mais difícil de carregar, entendeu? O pessoal já ta mais cansado, mais estressado. (E2)

Tanto o paciente que é o cliente, exige muito da gente, tanto o colega de trabalho, que gera estresse na gente em determinadas situações, e do paciente que às vezes vem com risco da vida dele e a gente tem que...depende muita coisa.(E7)

Conforme evidenciado nas falas citadas, são apontados como fatores que levam à sobrecarga de trabalho e geradores de estresse a falta de tempo adequado para a realização das atividades laborais exige que o trabalhador as realize com maior rapidez e muitas vezes sem o cuidado necessário, além da falta de profissionais capacitados no setor exigindo que o trabalhador seja escalado por vezes sucessivas para a mesma atividade.

Esta sobrecarga de trabalho provoca um estado de superestímulo no indivíduo, pois as exigências excedem a capacidade do trabalhador de processar ou cumpri-las, inviabilizando o melhor desempenho no trabalho, além de ser um preceptor de estresse, levando a danos à sua saúde física e mental (MEIRELLES; ZEITOUNE, 2003). Tal sobrecarga foi evidenciada como fator preditivo de estresse em um estudo realizado com 100 funcionários que trabalham em sala operatória em hospitais filiados a Isfahan University of Medical Sciences, onde apontou-se sobrecarga de trabalho, falta de fontes de apoio, os conflitos com os colegas, a mortalidade e a dor dos pacientes, a dúvida sobre o tratamento, os conflitos com os médicos e falta de conhecimento adequado, como fatores geradores de estresse (BAKHTIARI; MEHRABI; HASANZADEH, 2013).

Outra questão de fundamental importância relacionada à carga de trabalho é o fato de existir associação significativa entre a carga de trabalho da equipe de enfermagem e eventos adversos na assistência prestada aos pacientes atendidos pelos trabalhadores de enfermagem (MAGALHAES; DALL'AGNOL; MARCK, 2013). Este estudo corrobora com esta categoria já que mostra a associação entre a carga de trabalho e a presença de eventos adversos na assistência prestada pela equipe de enfermagem.

$\mathrm{Na}$ categoria Falta de planejamento das atividades, dos recursos humanos e materiais, as falas mostram que:

Frequência muito grande de cirurgias complexas, isso causa bastante estresse devido à falta de funcionários, de recursos humanos, temos bastante atestado e pessoas que aposentaram e não foram repostas, o déficit de funcionários que a gente tem é enorme, ou seja, todos estão bastante cansados estressados fazemos praticamente jornada dupla. (E3)

O ponto pior do nosso é esse estresse do dia a dia é pela falta de material, falta de funcionário, o doutor quer material que não tem, então isso gera muito desconforto. (E9)

Ah! Então! Por que envolve paciente, precisa de muito planejamento, trabalho de urgência. Aí você tem que se virar nos trinta para dar conta, para fornecer o que é necessário para cirurgia! Falta material! Então é estressante! Então quando o planejamento não é correto, a parte física sofre, porque além de você correr atrás de tudo que precisa, tem o estresse emocional do que você não consegue. (E13)

Você passa por algum estresse, fica brava porque às vezes tem uma cirurgia programada, depois não é aquela mais, de repente, você está numa sala e vai para outra, é assim, falta planejamento sempre. A falta de materiais e equipamentos também me estressa demais, fico que nem sei como dizer! (E15)

Apesar da correria, é um ambiente desorganizado. 
As falas evidenciam que a falta de planejamento das atividades a serem desenvolvidas, falta de materiais, de recursos humanos e de equipamentos, são fatores que os levam ao estresse. No que se refere à falta de planejamento das atividades a serem desenvolvidas, estudos demonstram que as incertezas e o desconhecido são apontados como fatores que geram medo e por sua vez levam o individuo ao estresse (CAREGNATO; LAURET, 2005).

Passos, Silva e Carvalho (2010) avaliando os fatores influenciadores de estresse entre os profissionais da equipe de enfermagem de um CC de um hospital particular da cidade de São Luís - MA, onde 35\% dos trabalhadores referem à falta de material e o acúmulo de atividades e responsabilidades e $12 \%$ referem à falta de recursos humanos como sendo fatores que levam ao estresse.

A falta de planejamento correto quanto à quantidade de materiais e equipamentos utilizados no CC pode desencadear conflitos entre a equipe multiprofissional, impossibilitando a assistência adequada (SILVA; POPOV, 2010). Outro ponto que também leva o trabalhador a ficar estressado é o trabalho e retrabalho causado pelo estabelecimento de um planejamento e organização não adequadamente delineados, porém observa-se que esta é uma característica da realidade dinâmica e imediatista do trabalho no centro cirúrgico.

Tem-se ainda que equipamentos e materiais que não funcionam ou que funcionam inadequadamente durante a cirurgia, uso de materiais inadequados ou ruins, falta de material e equipamentos em quantidade insuficiente para os pacientes, uso de artigos ou produtos impróprios e improvisos, são referidos como estressores para a equipe cirúrgica, sobrecarregando o desempenho do colaborador e gerando estresse. Estas situações vivenciadas como estressantes afetam de forma significativa o equilíbrio do profissional (CAREGNATO; LAUTERT, 2005; SILVA; POPOV, 2010).

$\mathrm{Na}$ categoria nomeada como Ambiente

fechado assim se expressam os entrevistados:

Como é um setor fechado muitas vezes se torna estressante a gente não vê luz, não vê outras pessoas, nossa comunicação com as outras pessoas é restrita e provoca uma angustia enorme. (E10)

Estar neste ambiente fechado, parece que estamos alheios ao mundo, isso é muito estressante. Não podemos nem nos comunicar com outros colegas de outros setores. (E8)

No meio desse ambiente, conturbado, é estressado porque é um ambiente fechado, cirurgias de grande porte. (E2)

Sabe-se que o CC é uma unidade fechada e com características próprias, onde há a necessidade de se restringir o contato pessoal de fora dessa unidade, não ocorrendo, portanto a relação interpessoal com as outras unidades da instituição. O contato com o ambiente externo normalmente ocorre por via telefônica e por meio do recebimento de materiais e recepção de paciente (SILVA; POPOV, 2010).

Passos, Silva e Carvalho (2010) apontam que o fato de os trabalhadores da enfermagem do CC realizarem as suas atividades laborais em ambientes fechados, o estresse pode ser um fator presente, já que esse tipo de setor faz com os trabalhadores se sintam excluídos de experiências e atividades que aconteçam no hospital, como a troca de experiências entre os trabalhadores e pouca participação em treinamentos levando muitas vezes à defasagem de atualizações sobre a prática nas suas atividades diárias.

No interacionismo simbólico, a relação do indivíduo com o ambiente de trabalho se dá pelo processo de socialização do mesmo com o meio onde se está inserido e com a forma como a sua personalidade se adéqua ao ambiente de inserção, já que em um ambiente de trabalho onde os atores se 
relacionam, a forma de adaptação do indivíduo ao ambiente vai facilitar ou dificultar as suas relações (CHARON, 1989).

Nas falas deste estudo evidencia-se que a interação dos indivíduos com o ambiente de trabalho pode gerar reações que predispõem ao desenvolvimento do estresse. A forma como cada trabalhador age no seu trabalho é o significado que o mesmo dá a esse trabalho, podendo este ser resignificado de acordo com o tipo de personalidade do trabalhador (DUPAS; OLIVEIRA; COSTA, 1997).

Se o trabalhador resignificar a sua forma de enxergar o seu trabalho, talvez ele mesmo possa encontrar formas de diminuir os geradores de estresse na sua atividade laboral.

\section{Conclusão}

Conclui-se que os profissionais de enfermagem vivenciaram o estresse relacionado com fatores de natureza objetiva como: a sobrecarga de trabalho, a falta de planejamento das atividades, dos recursos humanos e dos materiais e equipamentos e o trabalhar em um ambiente fechado.

Pode-se afirmar ainda que resultados desta pesquisa podem servir de subsídios para ampliar a qualidade de vida no trabalho da equipe de enfermagem de CC atuam em hospitais, visto que, a identificação dos estressores pode viabilizar ações para elencar estratégias de enfrentamento eficazes para lidar com o estresse no trabalho, com repercussões no desempenho, na saúde e na qualidade da assistência.

Embora os resultados da presente investigação demonstrem que os objetivos propostos foram alcançados, é imprescindível considerar os resultados em sua singularidade, uma vez que reflete a realidade de uma parcela de profissionais de enfermagem de um CC de um hospital universitário do sul do país.

\section{Referências}

BAKHTIARI, S.; MEHRABI,T.;HASANZADEH, A. An investigation on occupational stress of the operating room staffs in hospitals affiliated to Isfahan University of Medical Sciences and its association with some factors. Iranian Journal of Nursing and Midwifery Resaersh, Baqiyatallah University of Medical Sciences, Tehran, Iran, v. 18, n. 2, p. 101-4, 2013.

BARDIN, L. Análise de conteúdo. 6. ed. Lisboa: Edições 70, 2011.

BRANDÃO, D. E. C.; GALVÃO C. M. O estresse da equipe de enfermagem que atua no período perioperatório: revisão integrativa. Revista da Rede de Enfermagem do Nordeste, Fortaleza, v. 14, n. 4, p. 836-44, 2013.

CAREGNATO, R. C. A.; LAUTERT, L. O estresse da equipe multiprofissional na Sala de Cirurgia. Revista Brasileira de Enfermagem, Brasília, v. 58, n.5, p. 545-50, 2005.

CHARON, J. M. Simbolic interacionism: an introduction, on interpretation, on integration. New Jersey: Prentice Hall, 1989.

DUPAS, G.; OLIVEIRA, I.; COSTA, T. N. A. A importância do interacionismo simbólico na prática de enfermagem. Revista da Escola de Enfermagem da USP, São Paulo, v. 31, n. 2, p. 219-26, 1997.

MAGALHÃES, A. M. M.; DALL'AGNOL, C. M.; MARCK, P. B. Carga de trabalho da equipe de enfermagem e segurança do paciente: estudo com método misto na abordagem ecológica restaurativa. Revista Latino-Americana de Enfermagem, Ribeirão Preto, v. 21, n. esp., 2013.

MEIRELleS, N. F.; ZEITOUNE, R. C. G. Satisfação no trabalho e fatores de estresse da equipe de enfermagem de um centro cirúrgico oncológico. Revista de Enfermagem Escola Anna Nery, Rio de Janeiro, v. 7, n. 1, p. 78-88, 2003.

MINAYO, M. C. S. Pesquisa social: teoria, método e criatividade. 29. ed. Petrópolis: Vozes, 2010 . 
MONTANHOLI, L. L.; TAVARES, D. M. S.; OLIVEIRA, G. R. Estresse: fatores de risco no trabalho do enfermeiro hospitalar. Revista Brasileira de Enfermagem, Brasília, v. 59, n. 5, p. 661-5, 2006.

OLER, F. G; JESUS, A. F.; BARBOZA, D. B; DOMINGOS, N. A. M. Qualidade de vida da equipe de enfermagem do centro cirúrgico. Arquivo de Ciência e Saúde, São José do Rio Preto, v. 12, n. 2, p. 102-110, 2005.

PASSOS, J. B.; SILVA, E. L.; CARVALHO, M. M. C. Estresse no centro cirúrgico: uma realidade dos profissionais de enfermagem. Revista de Pesquisa em Saúde, São Luis, v. 11, n. 2, p. 35-8, 2010.

RIBEIRO, R. P. Prevalência da sindrome metabólica entre trabalhadores das equipes médica e de enfermagem de um hospital do Paraná e sua associação com estresse ocupacional, ansiedade e depressão. 2012. 160 f. Tese (Doutorado em Enfermagem) - Universidade de São Paulo, Ribeirão Preto-SP.

RODRIGUES, A. B.; CHAVES, E. C. Fatores estressantes e estratégias de Coping dos enfermeiros atuantes em Oncologia. Revista Latino-Americana de Enfermagem, Ribeirão Preto, v. 16, n. 1, 2008.

SANTOS, R. M. A.; BERESIN, R. Quality of life of nurses in the operating room. Hospital Israelita Albert Einstein, São Paulo, Brasil, v. 7, n. 2, p. 152-8, 2009.

SCHMIDT, D. R. C.; DANTAS, R. A. S.; MARZIALE, M. H. P.; LAUS, A. M. Estresse ocupacional entre profissionais de enfermagem do bloco cirúrgico. Texto e contexto - Enfermagem, Florianópolis, v. 18, n. 2, 2009.

SEMENIUK, A. P.; DURMAN, S.; MATOS, F. G. O. A. Saúde mental da equipe de enfermagem de centro cirúrgico frente à morte. Revista SOBECC, São Paulo, v. 17, n. 4, p. 48-56, 2012.

SILVA, P. P., POPOV, D. C. S. Estresse da equipe de enfermagem no centro cirúrgico. Revista de Enfermagem UNISA, São Paulo, v. 11, n. 2, p. 125- 
